\title{
Using Spatial Audio for the Enhanced Presentation of Synthesised Speech within Screen-Readers for Blind Computer Users
}

\author{
Kai Crispien, Wolfgang Würz ${ }^{2}$, Gerhard Weber ${ }^{3}$ \\ (1) Institut f. Fernmeldetechnik \\ (2) Institut f. Kommunikationswissenschaft \\ Technische Universität Berlin \\ (3) F.H. Papenmeier GmbH\&Co KG, Schwerte \& \\ Institut f. Informatik, Universität Stuttgart \\ email: crispien@ftsu00.ce.tu-berlin.de
}

\begin{abstract}
In order to enhance the presentation of synthesised speech output within GUI-based screen-readers for blind computer users, a spatial audio processing system, based on head-related transfer function processing technology is used to apply virtual acoustic positioning to synthesised speech. The spatial audio processing system and methods for generating spatial auditory displays are introduced. A procedure for the integration and synchronisation of an external speech synthesiser to the system is described. Finally, some prospects for the use of the enhanced speech output facilities of the system are given.
\end{abstract}

\section{Introduction}

Until recently, human-computer interfaces have consisted mainly of textual information represented by ASCII-coded characters. Blind computer users can access these command line-based interfaces by using a screen-reader software which usually reads the text displayed on the screen and provides information to either a synthetic speech synthesiser and/or a tactile text display, consisting of refreshable Braille modules. The introduction of graphics-based user interfaces, referred to as GUIs (Graphical User Interfaces), has raised various problems for blind computer users, since access to these human-computer interfaces requires enhanced methods and design of non-visual computer interaction.

One approach for enhancing the auditory representation within screen-readers for GUI systems is the spatial presentation of audio information, represented by auditory interaction objects, referred to as "auditory icons", or synthesised speech output $[1,2]$. The spatial presentation of audio information can provide orientation and navigation aids within the user interface, since the position of interaction objects, text components or pointing devices becomes determinable for non-visual users, exploiting the human perceptual ability of auditory localisation. 
For the representation of continuous text, appearing for example in word processing or spreadsheet applications, spatial positioning of synthesised speech output can enhance the immediate understanding of the text layout and enables improved interaction methods. Additionally, non-verbal audio components can be used to identify text attributes and to assist pointing in text-based applications.

\section{GUI-based Screen Readers}

Blind users have learned since nearly a decade to work with speech synthesisers for access to text-based application programs running on PCs. Especially in North America work places for blind users have been equipped with a synthesiser and a screen reading program (or simply called screen-reader). In contrast, Braille displays are read actively by the fingers while speech output allows the user to be passive.

For access to graphical user interfaces a verbalisation of the graphical interaction objects like windows, menus, buttons, icons has to be generated by a dedicated screen-reader program. The screen-reader relies for this on a model of the visual interaction objects which is called an off-screen model (OSM, see [3]). While an OSM is hierarchically structured such a model can also consist only of lines of text and is flat. We then call this model a virtual screen copy (VISC).

The screen reader handles two activities:

- tracking of changes on the screen and reporting about these changes through appropriate verbalisation to the user

- assist the user in review the contents of the screen (receptively the OSM or VISC)

The spatial information describing the size, position, overlapping of interaction objects can be presented on a large Braille display appropriately (see [4]). For speech-based screen readers current solutions only make use of the hierarchical model of an OSM. During tracking the screen reader selects one node in the OSM which is active. During review the user traverses through this hierarchy in order to gain an overview on the windows contents or the complete screen. The spatial relation among interaction objects is currently not properly communicated, res. absent in the OSM. As long as interaction remains keyboard based such an approach can be suitable but many applications are operated more easily with a mouse or other pointing devices. Recent research has found that pointing is also suitable for blind users if non-verbal acoustic feedback is used (see [5]). In order to provide an understanding of the spatial relations within the user interface it is proposed to apply a spatial acoustic representation to verbal and non-verbal auditory interaction components. This, can improve for example the use of pointing devices since positions of interaction objects become determinable through direct perception of acoustic locations. 


\section{$3 \quad$ Spatial Audio Processing}

Conventional stereophonic recordings, monitored with headphones, only provide a relatively poor auditory locational performance. The perceived position of a sound source usually appears along an axis through the middle of the head. This phenomena is known as the so-called "inside-the-head" localisation [6].

This is based on the fact, that even though interaural temporal and intensity differences (ITD, IID) appropriate to an external source are present, the directiondependent reflection and refraction effects of the head, outer ear (pinnae) and shoulders are absent in the recording. Many studies suggested the importance of these effects, acting as a direction-dependent frequency filter within the human auditory system. Especially for the determination of sound sources in the median plane (in front, above or behind of the listener), where ITDs and IIDs are not present, these spectral distortions of the head and body provide the primary cue for the human auditory localisation system [6]. Therefore, an appropriate model of the human acoustic system, such as a dummy-head with microphones embedded in its artificial ears, can simulate a close approximation of the natural listening impression within a three-dimensional spatial sound field. Providing an improved externalisation or outside-the-head listening impression, if monitored with headphones.

With the introduction of powerful signal-processing technology in the 1980s, a technique for the computational simulation of spatial sound presentation with headphones was investigated (see e.g. $[7,8]$ ) and led to the development of a number of real-time processing systems. In general, these systems use a collection of head-related transfer functions (HRTFs), usually derived from measurements of a sound stimulus placed in certain appropriate directions around a listener. The stimuli are measured with probe microphones placed in the outer ear of either individual human subjects or dummy-heads, resulting in a pair of specific impulse responses for each direction. These HRTFs then can be convoluted with an audio signal in the time-domain, performing as direction-dependant filters. In order to achieve real-time performance, finite-impulse-response (FIR) filters are used for the fast convolution process. HRTF processing thus represents a linear system that is a function of sound source position.

Applying this processing method to an audio signal, monitored with headphones, produces a perception of spatially located sound sources in a virtual threedimensional acoustic free-field ${ }^{1}$. Motion of sound sources can be achieved by interpolation between certain transfer functions. Head motions of the listener, which form another important cue used by the human auditory system, can also be integrated in the simulation process.

\footnotetext{
${ }^{1}$ An acoustic free-field is defined as an reflection-free acoustic field. A sound source is not superimposed by any sound waves reflected from boundary surfaces, like in a reverberant room (diffuse-field).
} 
Therefore, the head position has to be transmitted and updated continuously using a "six-freedom-of-choice" position-tracking device mounted on top of the headphone. Present systems offer different numbers of channels that can be independently spatialised for azimuthal and elevational directions. These systems are used so far in aviation and aerospace applications [9], enhanced radio-communications, musical recording and mixing [10] and auditory displays for man-computer interaction [11].

\subsection{The Beachtron ${ }^{\mathrm{TM}}$ HRTF-based Audio Processing System}

Due to the fact that HRTF processing, carried out in real time, requires extensive processing load, systems available so far have been highly cost-intensive and not affordable for applications in the rehabilitation area. Only recently, in 1993 a costeffective system became available on the market, namely the "Beachtron" system from the pioneering American manufacturer Crystal River Engineering, Inc..

This system combines a high quality digital sound processing device for PCM recording and playback of sounds and a musical instrument synthesiser with a HRTF processing-engine. Additionally, external audio sources can be applied to the system.

The Beachtron system consists of a ISA bus-based plug-in board which is capable to process two independent sound sources in real time. The boards are cascadable, thus up to sixteen individual spatialised sound sources can be presented simultaneously. Monitoring is carried out with a conventional, high quality headphone (Sennheiser HD540). Except the headphone, no additional external hardware is required for the generation and spatialisation of sounds.

The system uses the processing method described before (figure 1). HRTF filters are obtained from measurements of one female subject (subject SDO) which participated in the experiment of Wightman and Kistler, 1989 [see 7, 8]. This subject showed the most accurate performance for either free-field or simulated headphone-based localisation of sound sources among all participants in the experiment. It was found that different persons are able to adapt to this subject's HRTFs and can perform acceptable accurate sound localisation[12].

The distance of sound sources is simulated by an "atmospheric absorption" model which attenuates higher frequencies. The degree of attenuation depends on the distance through which the sound travels in the atmosphere. This results in a lowpass-filtered or "muffled" characteristic of distant sound sources. Motion of sound sources, res. head motions - monitored by an additional position-tracking device, are processed by using an interpolation algorithm between the four nearest stored transfer functions.

\footnotetext{
${ }^{\mathrm{TM}}$ The Beachtron is a registered trademark of Crystal River Engineering, Inc., 12350 Wards Ferry Road, Groveland, CA 95321, USA; Phone: (209) 962-6382, Fax: (209) 962-4873.
} 


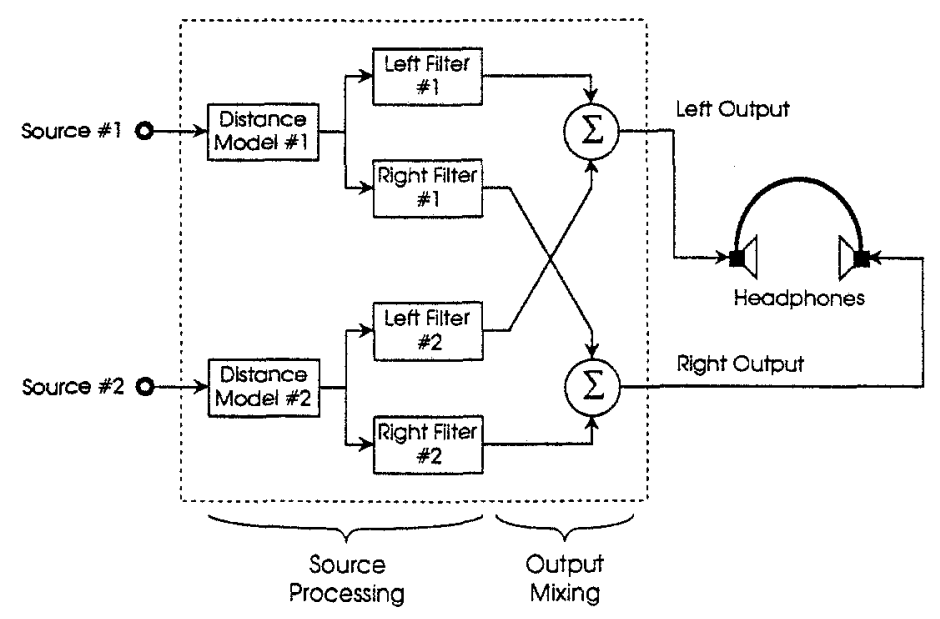

Fig. 1: Spatialisation processing in the Beachtron system.

\subsection{Generation of a Spatial Auditory Display with the Beachtron System}

In order to generate a spatial auditory representation of the computer screen with the Beachtron system, such as the auditory part of a screen-reader, auditory objects can be generated by attaching a specific sound representation and an auditory position to a screen object. Sound representations can be either any PCM waveform sound $(8 / 16$ bit, $44.1 \mathrm{kHz})$, a preset-based synthesiser sound or synthesised speech, generated by an external speech synthesiser. The position of an auditory object within the virtual acoustic free-field is described by a three-component vector $[\mathrm{x}, \mathrm{y}, \mathrm{z}]$ (see figure 2).

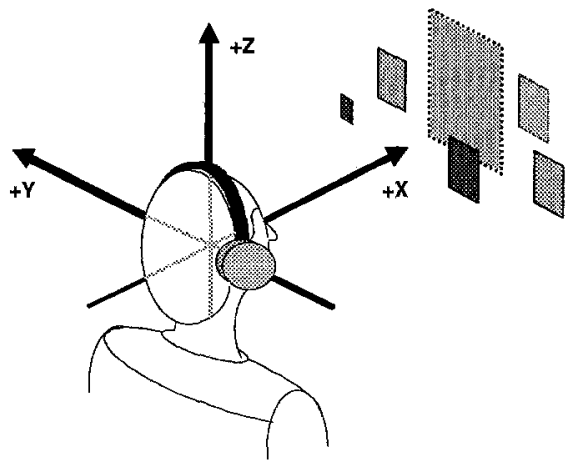

Fig. 2: Conceptual illustration of a spatial auditory display.

Due to the fact, that such a virtual spatial representation of auditory objects is not restricted to physical dimensions (as it would be the fact, if monitored with a loudspeaker system), a spatial mapping between the visual representation of the GUI (computer screen) and the auditory representation can be carried out which is suitable for an optimised representation of the auditory display. 
Therefore, a geometrical projection has to be applied which transforms the 2D rectangular screen-position into a virtual 3D auditory position. In order to make maximum amount of the facilities of the human auditory localisation system - which does not perform linear for all directions [6], an extended projection can be chosen. A projection of the screen plane to an cylindrical shape seems to be a good mapping, since the elevational plane, where sounds are usually localised more inaccurately [6], can be extended above and under the listener's position.

In our approach, currently mainly positions on the frontal hemisphere of the listener are used for the mapping of screen contents to the auditory display. The back hemisphere can be used for the presentation of extraordinary events in the humancomputer interface, like e.g. on-line help information. The third dimension (X plane) of the auditory display, appearing as acoustical distance information, can be used to represent background processes or inactive interaction objects, e.g. a file transmission process or overlapped windows.

\section{$4 \quad$ Enhanced Representation of Synthesised Speech}

\subsection{Spatial Representation of Synthesised Speech}

In general, speech synthesisers devices provide speech output by text-to-speech conversion of ASCII-coded character strings. Textstrings, usually transmitted to the device via the serial port, are examined for phrase and word components which can be spoken, res. commands for the control of the device. Common control parameters are: speech timbre, pitch, speed, word prosody, etc..

Although, a larger number of speech synthesisers are available only a few devices supply multilingual text-to-speech conversion. A well known device in Europe is for example the "CompactVoxBox" by the Swedish manufacturer Infovox. This device offers text-to-speech conversion for up to ten different European languages. Additionally, the CompactVoxBox provides a software-based facility, called indexing which enables the possibility of sending index commands to the text-tospeech converter at specific locations in the text which are transmitted back to the screen-reader. Therefore, indexing can be used for the control of the asynchronous behaviour of the speech synthesiser within an application program. An index-string consist of an index-identifier (e.g. ASCII 'ctrl-A') and an index-marker consisting of a three-position digit (e.g. '001'). If a textstring is passed to the speech synthesiser, containing one or multiple index-strings, the text-to-speech unit returns the index-markers on the serial port during speech output. The index marker is transmitted back immediately after the succeeding regular text part has been spoken.

In order to achieve spatial representation of the speech output facilities of a screen reader the speech synthesiser is applied to the external input connector of the Beachtron board. 
Static text components, such as window or icon titles, menu text, etc., can be spatialised by attaching their screen position to the Beachtron system before the specific text string is transmitted to the speech synthesiser via the serial port. The incoming synthesised speech signal is then processed spatially and appears at the specified acoustic position.

For the spatial representation of continuous text, like. e.g. text edited in a word processor, the spatial acoustic position of the text position has to be updated continuously. This requires a mechanism which synchronises the speech synthesiser to the spatial acoustic processing system. Therefore, a string handling procedure concatenates text parts (e.g. single words or characters) delimited by white-space characters (e.g. blanks, tabs, etc.) with an index-marker. These index-markers contain the column position (e.g. 1-80) of the specific text parts (see figure 3 ).

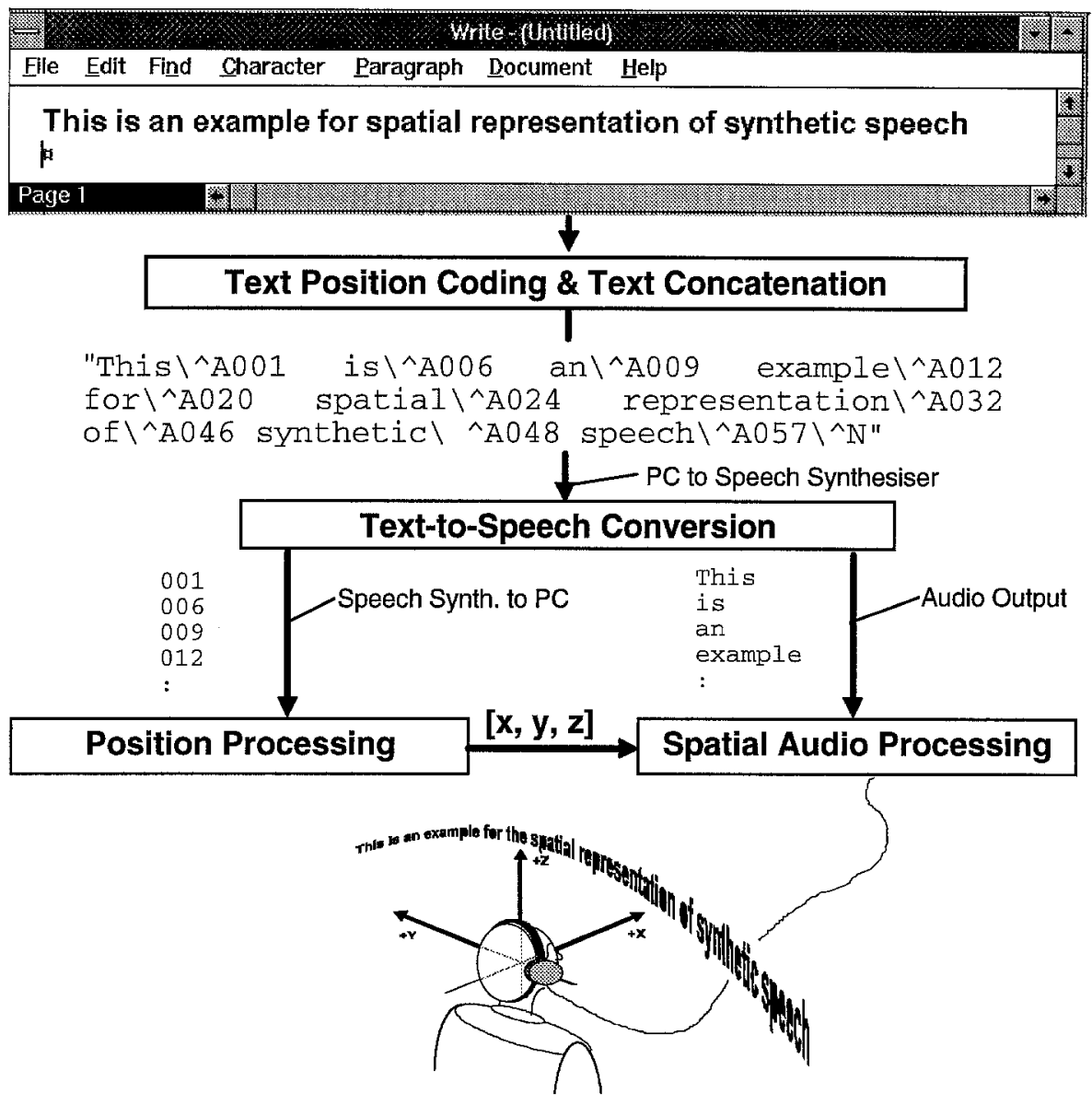

Fig. 3: Mechanism for the spatial representation and synchronisation of synthesised speech. 
The concatenated text string is then passed to the speech synthesiser and processed by the text-to-speech unit. If an index-marker is returned from the text-to-speech unit the index-marker, contains the column position of the successive text part which is used for the computation of the spatial acoustic position of the speech output.

Beside the spatialisation of text, also the asynchronous behaviour of the speech synthesiser is taken into account, if for example the speed of the speech output is slowed down by the user. The mechanism therefore provides a synchronised, closedloop between the speech synthesiser and the spatial acoustic processing system which updates the azimuthal acoustic text position continuously (figure 3). Vertical positions of different lines within the text are processed by using the line-feed character (ASCII ' $\mid n$ ') as an incriminator for the elevational acoustic positions.

\subsection{Interaction with Spatially Represented Speech}

Spatial representation of speech output the assists the user to identify the position of spoken text parts in relation to the visual representation on the screen. This can enhance the immediate understanding of the text layout within a word processor and other text-based applications. An example for the use of this information is the representation of multi-column text as used in documents, tables or spreadsheets.

The synchronisation between screen and audio-output has some useful implications, especially in the combination with the use of pointing devices. On a technical level, it is now possible to synchronise the caret cursor or mouse pointer and the acoustic speech-position.

During tracking of the mouse pointer movements, spoken feedback can be handled in two different ways (see [13]):

- the currently spoken item is interrupted; a new item is pronounced immediately when reached

- the currently spoken item is completed; instead of speaking a new item a nonverbal sound is produced

Synchronisation of the speech output with pointing enables the user not only to follow passively the screen-reader, but provides the opportunity to interact actively with the text components through pointing.

Additionally, non-verbal sounds can be controlled by the synchronisation mechanism. Non-verbal sound components then can be used for conveying additional information during text-based interaction as for example text attributes like bold, italic, underlined, etc. text styles or highlighted text, commonly used in hypertext applications (such as e.g. the MS-Windows help facility). 
Therefore, one digit of the index-maker can be used for the coding of specific text attributes within the indexing mechanism, described in section 4.1 .

A typical scenario for a hypertext-based interaction with the system is for example, that a non-verbal sound is started if a highlighted text part is read by the speech synthesiser. This "hypertext-sound" keeps on playing in the background until the speech synthesiser has finished reading the highlighted text part. Through the presentation of the non-verbal sound in parallel to the speech information the user becomes able to monitor the specific highlighted text information and to navigate in the hypertext system by activating the hypertext through keyboard- or pointing device-based interaction.

\section{$5 \quad$ Conclusions}

This paper has proposed a method for the enhancement of synthesised speech output within GUI-based screen-reader programs. Synthesised speech is represented at within a virtual spatial auditory display, processed by a spatial audio-processing system. The location of the spatially represented speech are computed from text positions derived by the screen-reader program. The mechanism also provides a synchronisation between the screen-reader and the asynchronous speech output. This can assist pointing and the control of non-verbal audio information for the communication of text attributes, which can enhance text-based interaction for blind computer users.

\section{Acknowledgements}

This work has been supported by the GUIB project ("Textual and Graphical User Interfaces for Blind Computer Users"), a pilot project of the EEC program TIDE ("Technology Initiative for the Disabled and Elderly"). Thanks to Prof. Dr.-Ing. Klaus Fellbaum and Andreas Korte at Technical University of Berlin for their support.

\section{References}

[1] Edwards, A.,\& Mynatt, E.: Mapping GUIs to Auditory Interfaces. UIST '92: The Fifth Annual Symposium on User Interface Software and Technology, Monterey, California, ACM Press, New York, 1992.

[2] Crispien, K.,\& Petrie, H.: Providing Access to GUI's Using a Multimedia System - Based on Spatial Audio Presentation. Audio Engineering Society 95th.Convention Preprint, New York, Oct. 1993.

[3] Schwerdtfeger, R.S.: Making the GUI talk. BYTE, Dec. 1991, pp.118-128. 
[4] Mynatt, E.;\& Weber, G.: Nonvisual presentation of graphical user interfaces: contrasting two approaches. in Proceedings of CHI'94, ACM Press, New York, 1994.

[5] Petrie, H., Heinila, J.,\& Ekola, H.: A Comparative Evaluation of Computer Input Devices for Blind Users. in Proceedings of ECART 2, Stockholm, Sweden, May 26 - 28, '93, ISBN 91-88 336/19/0, 1993.

[6] Blauert, J.: Spatial Hearing: The Psychophysics of Human Sound Localizaton. Cambridge, MA, MIT Press, 1983.

[7] Wightman, F. L.,\& Kistler, D. J.: Headphone Simulation of Free-Field Listening. I: Stimulus Synthesis. J. Acoustic Society of America, Vol. 85, Febr. 1989, pp. 858 - 867, 1989.

[8] Wightman, F.L,\& Kistler, D.J.: Headphone Simulation for Free-Field Listening II: Psychophysical Validation. J. Acoustic Society of America, Febr. 1989, pp. 868-878, 1989.

[9] Wenzel, E. M.: Three-Dimensional Virtual Acoustic Displays. in Blattner, M.M., \& Dannenberg, R.B., (eds.): Multimedia Interface Design, ch. 15, pp. 257 - 288, ACM Press, New York, 1992.

[10] Gierlich, H. W.,\& Genuit, K.: Processing Artficial - Head Recording. J. Audio Engineering Society, Vol. 37, No. 1/2, 1989.

[11] Ludwig, L., Pincever, N.,\& Cohen, M.: Extending the Notion of a Window System to Audio. Computer, 23, pp. 66 - 72, 1990.

[12] Wenzel, E. M., Arruda, M., Kistler, D. J.,\& Wightman, F.L.: Localization Using Nonindividualized Head-Related Transfer Functions, $\underline{\text { J. Acoustic }}$ Society of America, July 1993, pp. 111-123, 1993.

[13] Weber, G.: Modelling interaction of blind people using graphical user interfaces. Vogt, F.H. (ed.): in Proceedings of 12th World Computer Congress, Personal Computers and Intelligent Systems. Information Processing '92, Elsevier Science Publishers B.V.(North Holland), pp. 39-46, 1992. 